\title{
Cracks avoidance with a modified solid tungsten divertor in ASDEX Upgrade
}

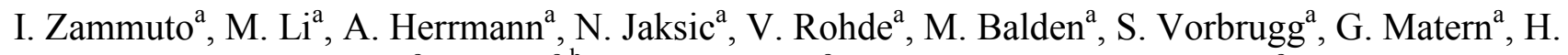 \\ Greuner $^{\mathrm{a}}, \mathrm{R} . \mathrm{Neu}^{\mathrm{a}, \mathrm{b}}$, A. Kallenbach ${ }^{\mathrm{a}}$, the ASDEX Upgrade Team ${ }^{\mathrm{a}}$ \\ ${ }^{a}$ Max-Plack-Institut für Plasmaphysik, D-85748, Garching, Germany \\ ${ }^{b}$ Technische Universität München, D-85748, Garching, Germany
}

To clarify the suitability of solid tungsten as plasma facing material, a new divertor, DIV-III, entered in operation in ASDEX Upgrade (AUG) in 2014. Since the first experimental campaign, AUG divertor tiles faced crack issues. Trying to identify the reasoning, a new set of tiles was installed in 2016: many precautions were taken to reduce the stresses possibly causing the cracks. The AUG campaign 2017 started in March and the first results were collected during the short summer opening in 2017. This paper reports on the modification of the tile setup, based on Finite Element Modelling, and on the progress achieved in the first operation phase.

Keywords: ASDEX Upgrade, tungsten divertor, tungsten heavy alloy, tungsten crack.

\section{Introduction}

According to the 'EFDA roadmap for the realization of fusion energy' the material portfolio for DEMO will consist of consolidated developments of EUROFER as a structural material and tungsten as plasma facing material. Bearing that in mind, AUG is presently equipped with ferromagnetic tiles in the inner column [1] and massive tungsten tiles in the strike line region of the lower outer divertor [2]. The remaining wall elements are made of tungsten coated graphite. The divertor III (DIV-III) is an adiabatically loaded component and each sector consists of a water cooled stainless steel support structure on which 8 massive tungsten tiles $(220 \times 75 \times 15$ $\mathrm{mm}^{3}$ ) are clamped (Fig. 1).

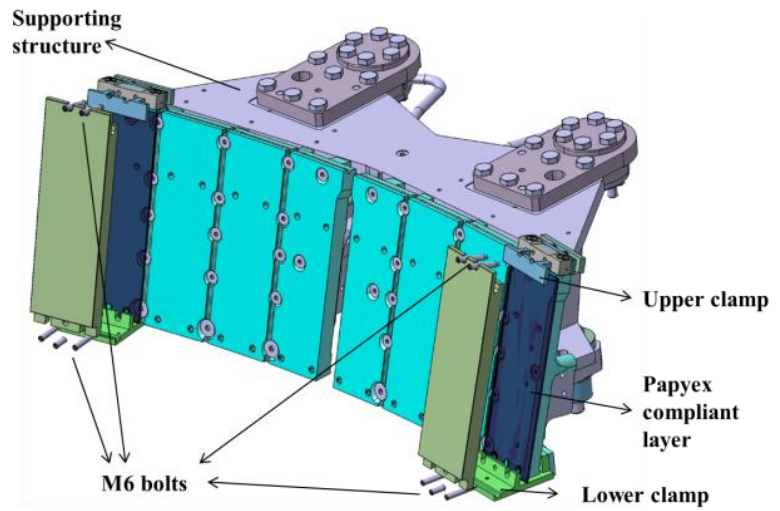

Fig. 1. Overview of one sector of the DIV-III: only the tiles in the outermost position are illustrated.

Before installing the new DIV-III, extensive studies, including thermo-mechanical Finite Element Modelling (FEM) of the tungsten tile [3-5] and qualification in the high heat flux test facility GLADIS [6], were carried out. After an experimental campaign, in-vessel inspection reveals shallow and deep cracks in most of the tiles [7]. Such cracks were not found after the high heat flux tests in GLADIS [6] and during prototype tests in AUG [3]. The damaged tiles did not jeopardize the machine operation, but to understand the origin of the phenomenon responsible for the crack, during the 2016 opening, modified tiles were installed.

This paper describes the reasoning behind the new divertor tile optimization and its first outcomes. Section 2 introduces the issues raised in the first AUG experimental campaigns. Section 3 describes how the crack issue can be tackle by means of FEM analyses. The tile acceptance tests and the divertor setup are given in Section 4. The preliminary outcome from the summer inspection is presented in Section 5. Finally, discussion, summary and further steps are presented.

\section{Findings from first campaigns and outline of the tile loads}

The tungsten evolution of AUG over the years and an extensive overview of the results collected during the first campaigns with a solid tungsten divertor in AUG are given in [2]. The first campaigns revealed two main findings, which can be summarized as follow: 1) shallow cracks in the strike line region of the tiles and 2) deep cracks going through the tile thickness and in line with the longer (poloidal) direction of the tile. In Fig. 2 the typical crack patterns are illustrated: deep crack goes along the tile length, connecting the clamp region with the middle one, and it runs through the entire tile thickness $(15 \mathrm{~mm})$. Conversely the shallow crack consists of a network of small and superficial cracks, with a depth of the order of hundred $\mu \mathrm{m}$. At first glance, the thermo-mechanical stress seems to be responsible of the deep cracks: during the plasma operation, a compressive plastic deformation is generated in the strike line region. In the cooling phase of the tile, large residual tensile stresses are induced by plastic deformation acting as the driving force of the opening of the deep cracks. The initiation of the cracks can be attributed to plastic fatigue or to the repeated and concentrated load derived by the edge localized modes (ELMs). On the other hand, the tiles tested in GLADIS 
did not show any thermoplastic effect in the benchmark phase of the DIV-III. But GLADIS HHF tests have shown that the crack can easily propagate in a precracked tile by moving the heat load towards an uncracked region.

Actually during the plasma operation, disruption events stress the tungsten tile, and they could be particularly damaging in an early operational phase, when the material is still cold. The disruption event induces eddy currents in the bulk material that, interacting with the static toroidal field, is responsible of moments acting into the components. Complex induced current paths between the stainless steel support and the tungsten tiles are also responsible for additional forces acting on the tungsten tiles. This 'bypassing current' is due to the low electrical resistivity of the tungsten with respect to the stainless steel of the supporting structure. The temporal variation of the radial magnetic field of $250 \mathrm{~T} / \mathrm{s}$ and the static magnetic field of $2.5 \mathrm{~T}$, typical values for AUG disruptions, cause a force of about $6.4 \mathrm{kN}$ for the outermost tiles. Such electromagnetic loads end up in a high stress concentration in the clamped regions of the tile.

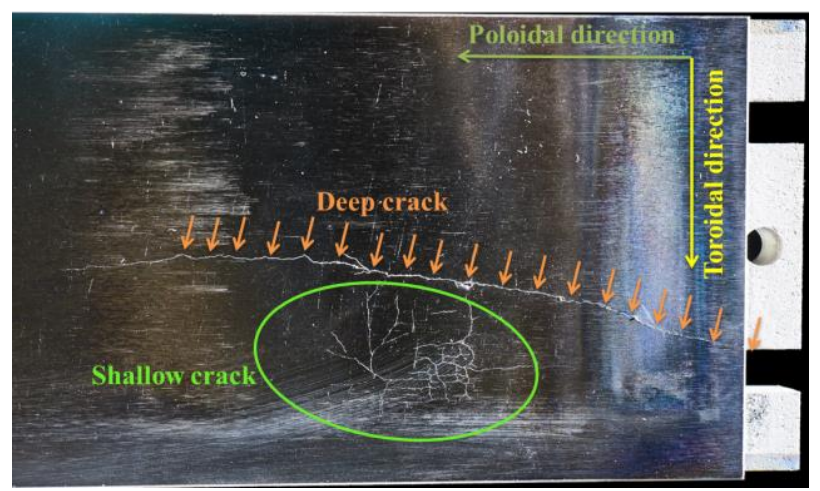

Fig. 2. Typical crack pattern in a tungsten tile: shallow cracks are identified by a dense superficial network, while the deep crack runs on poloidal direction from the lower part (right side) of the tile to the central one extending through the entire tile thickness.

The extensive thermo-mechanical and electromagnetic FEM modeling performed to explain the deep cracks did not reveal an unambiguous mechanism of crack initiation and propagation [7]. To remove this uncertainty, the divertor tiles were modified according to a new set of FEM analyses where the thermo-mechanical stress was reduced and the flexibility of the tile clamp was enhanced, both independently and combined.

Besides the FEM optimization, a further study of tungsten heavy alloys is envisaged, in particular of the tungsten $\mathrm{Ni} / \mathrm{Fe}$ heavy alloy (HPM 1850 from $\mathrm{HC}$ Starck). A comprehensive overview of the pros and cons of using a tungsten heavy alloy in fusion application, together with the qualification carried out in $\mathrm{AUG}$, is given in [8].

\section{Actions taken to reduce the occurrence of deep cracks}

\subsection{Optimization of tile shape}

To reduce the thermo-mechanical stress in the loaded region, the toroidal dimension of the tungsten tile should be reduced [9]. Keeping the same clamping design, two possibilities were considered: 1) split the original tile (wide tile) in two tiles with half toroidal width (split tile) or 2) introduce deep castellations along the poloidal direction.

The residual stress component, which is normal to the plane of the crack, of a wide and a split tile are compared in Fig. 3.

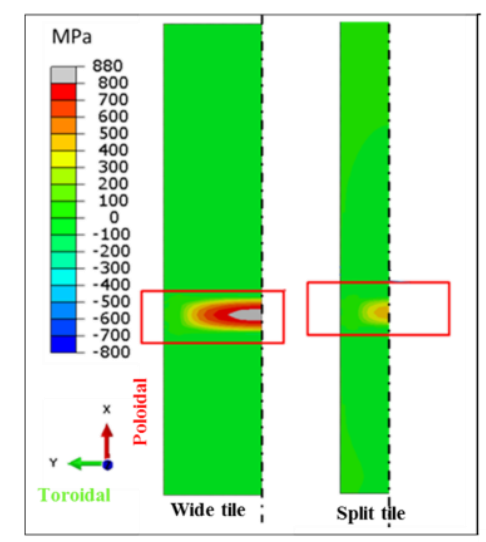

Fig. 3. Residual stresses in toroidal direction after cooling down: comparison between the wide and split tile. The dashed lines on the right side indicate symmetry axis used in the FEM analyses.

The FEM analyses refer to a heat load of $15 \mathrm{MW} / \mathrm{m}^{2}$ for $2.5 \mathrm{~s}$ for both cases. A strong reduction of plastic deformation and, consequently, of the residual stress along the toroidal direction after cooling down is observed in split tile compared to wide tile. The maximal tensile stress after cooling down at the surface is 880 $\mathrm{MPa}$ and $482 \mathrm{MPa}$, for the wide and split tile, respectively. A stress reduction is calculated also in case of a double poloidal groove or castellation on top of the tile surface, but a minimum depth of the castellation is required to avoid stress concentration in the castellation tip. For a moderate castellation depth, the stress increases, but from a depth of $6 \mathrm{~mm}$ on a reversal of the trend is found as shown in Fig. 4. The tile with double castellations, with $10.5 \mathrm{~mm}$ of castellation depth, has a residual stress of $866 \mathrm{MPa}$ after the cooling down, which is further reduced to $700 \mathrm{MPa}$ with the edge smoothing of the grooves. A dedicated paper on the thermomechanical analysis will be published elsewhere [10].

\subsection{Enhancement of the flexibility of the tile clamping and reduction of the bypassing current}

Nearly all deep cracks are connecting poloidally the region of high heat load with the lower part of the tile, where it is clamped to the support structure. A careful check of the tiles exposed in the previous campaign 
discloses a couple of tiles having the cracks just on the lower part.

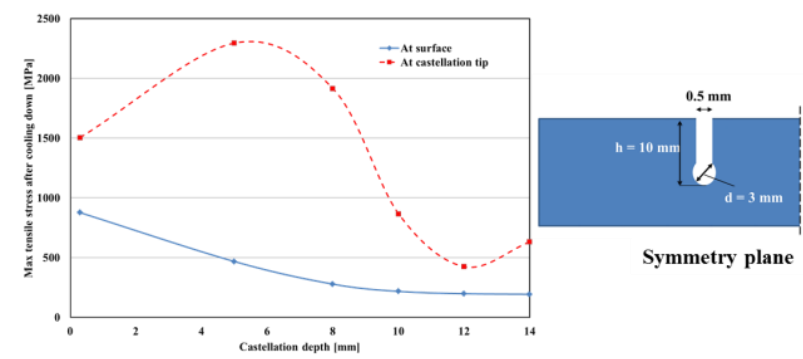

Fig. 4 On the left hand side: maximum residual stresses in toroidal direction after cooling down for the wide tile with double castellation. The solid line indicates the residual stress on the tile surface; the dashed line indicates the residual stress at the castellation tip. On the right hand side the castellation dimension of built tiles are reported.

Based on these evidences, the crack could be affected by the rigid constraint of the stainless steel clamps that, at least in the lower part and in combination with the loads arising during a disruption, could end up in a sudden and peaked stress distribution into the tile. To relax this contact stress, the steel clamp is replaced by a more elastic titanium alloy, so that the tile can have some freedom in its movements.

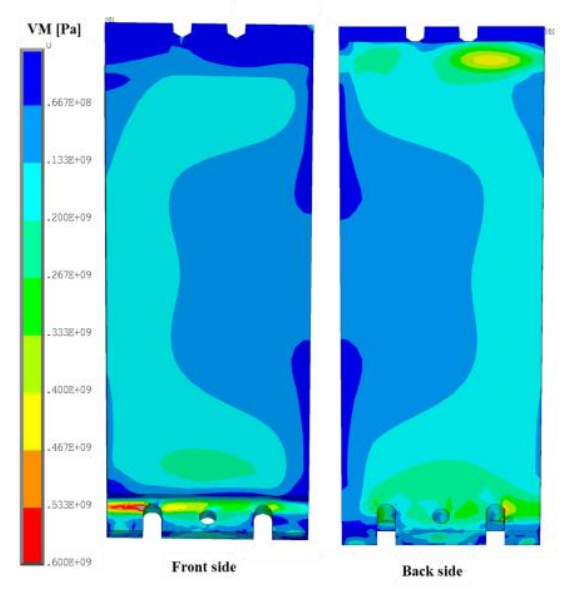

Fig. 5. Von Mises stress $[\mathrm{Pa}]$ distribution in the tungsten tile with titanium clamp during a disruption.

In Fig. 5 the stress distribution for the titanium clamp is illustrated. With similar forces acting on the tile, a stress relaxation of about $10 \%$ is expected moving the peak stress from $875 \mathrm{MPa}$ to $760 \mathrm{MPa}$ using a titanium clamp. For the eddy current calculations a dB/dt of $250 \mathrm{~T} / \mathrm{s}$ and a static toroidal field of $2.5 \mathrm{~T}$ are considered. In addition, the higher electrical resistivity of titanium with respect to the austenitic steel reduces the current bypassing through the tungsten tiles, with a corresponding reduction of $25 \%$ of the load acting on the tile. Therefore, the tiles installed with titanium clamps will benefit from a double effect: reduction of electromagnetic load and some more flexibility.

\section{Acceptance tests and divertor setup}

The manufacturing of the tungsten tiles is entrusted to two companies: Osnabruegge and MGS Precision. Before mounting, all the tiles were visually inspected and about $10 \%$ of them were examined with fluorescent dye penetrant. Small imperfections of the superficial condition of the tiles are recorded for later comparison. Macroscopic differences are highlighted between the two suppliers, but they are not confirmed microscopically.

For each supplier, one tile has been high heat flux tested in GLADIS, within the standard qualification procedure for AUG (200 cycles with $10.5 \mathrm{MW} / \mathrm{m}^{2}$ for $3.5 \mathrm{~s}$ ). Both tiles passed the test without any sign of damage or surface modification. Aware that the induced current depends on the tile position inside a sector, the divertor setup was organized so that the tiles of two suppliers are equally distributed inside the vessel. In this way, a strict comparison of the two material qualities can be done.

Following the optimization of the thermally induced stress, while keeping the manufacturing process as simple as possible, the solution of the split tile is selected as the favorite. As drawback, mounting the split tiles inside the vessel is more complicated than for the wide tile. At 6 out of 8 positions in 13 out of 16 divertor modules tungsten split tiles were installed with stainless steel clamps. The remaining two outermost positions were used to install more ductile tungsten heavy alloy, since in this position the highest bypass currents are expected. In Fig. 6 a CAD model of the standard sector setup is shown.

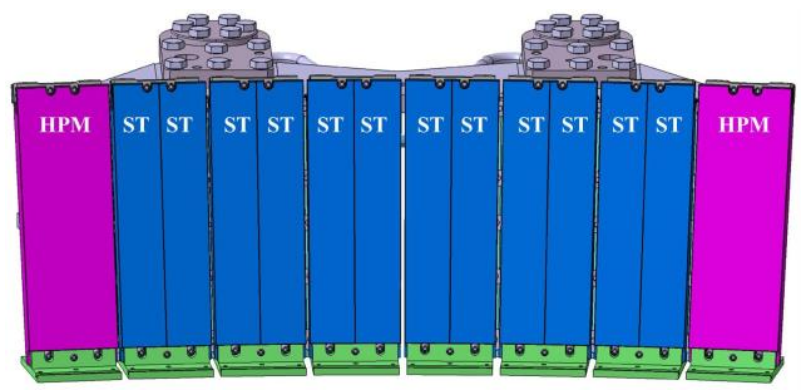

Fig. 6. CAD model the standard module installed in AUG. The outermost pink tiles are made of HPM and the remaining are the split tungsten tiles.

To disentangle the thermo-mechanical stress from the stress related to the rigid clamping arising during a disruption, special setups of tungsten tiles are installed in 3 divertor sections. To verify how the stiffness of the tile clamping affects the crack initiation/propagation, in one divertor module (sector 7) the original wide tile design is kept and the stainless steel clamps were replaced by the titanium clamps (see Fig. 11). In a second module (sector 15), the split tiles were installed together with the titanium clamps, combining both optimizations. Finally, in a third module (sector 16) tiles were installed that were fully optimized with respect to thermo-mechanical properties, where no plastic strains are expected, according to the FEM. These tiles are castellated in poloidal direction with two equidistant cuts of $10 \mathrm{~mm}$ 
depth and $0.5 \mathrm{~mm}$ width. The castellation was done via spark erosion process. In these three special sectors, the tungsten heavy alloy is not mounted, allowing a direct comparison with the old AUG divertor setup (wide tiles and stainless steel clamps). The divertor setup is summarized in Fig. 7. It should be mentioned that none of these solutions fit with the tiles embedding the Langmuir probes [12]. Distributed holes along the poloidal extension make the tile prone to cracks failure. For the next campaign this special tile should be redesigned for this application.

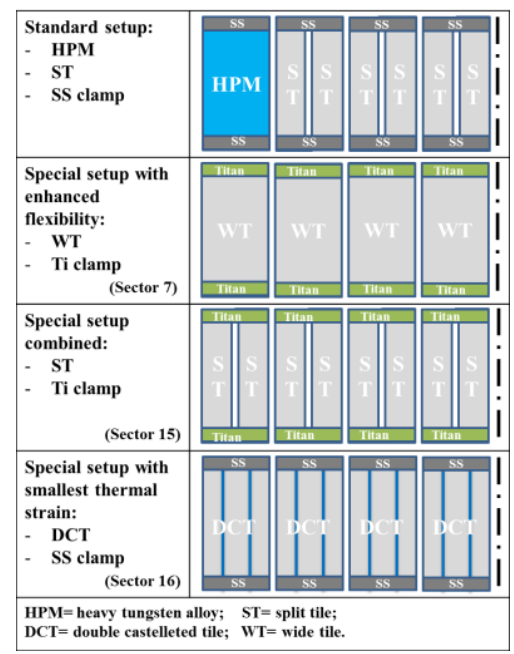

Fig. 7 Summary of the divertor setup: 13 sectors are equipped with the standard configuration. One sector for each special configuration is installed. All these setups are mirrored with respect to the dashed lines indicating the symmetry axis.

\section{Outcome of first inspection}

The AUG operation started in March 2017 and a short summer opening allowed the inspection of the newly installed tiles. The DIV-III stands about 760 shots, most of them in lower single null configuration and about half of them end up in a disruption. The operation ranged over the first part of the experimental campaign is reported in Fig. 8. Unfortunately, the operation of AUG suffered during last campaign from cracks in the above mentioned tiles embedding Langmuir probes, namely in sector 8 and sector 13. One of the two tiles built up a deep crack that running all along the toroidal width, split the tile in 2 half (see Fig. 9). Thus, the cooling rate temperature of the tile was drastically reduced, causing a prolongation of the dwell time between shots.

The replacement of the broken tiles gave the possibility to inspect the whole DIV-III. For the first time, since AUG moved to bulk tungsten as plasma facing component, no deep cracks were found in all sectors of the new installed tiles. Fig. 10 illustrates that both the split tiles and the castellated tiles are free of cracks.

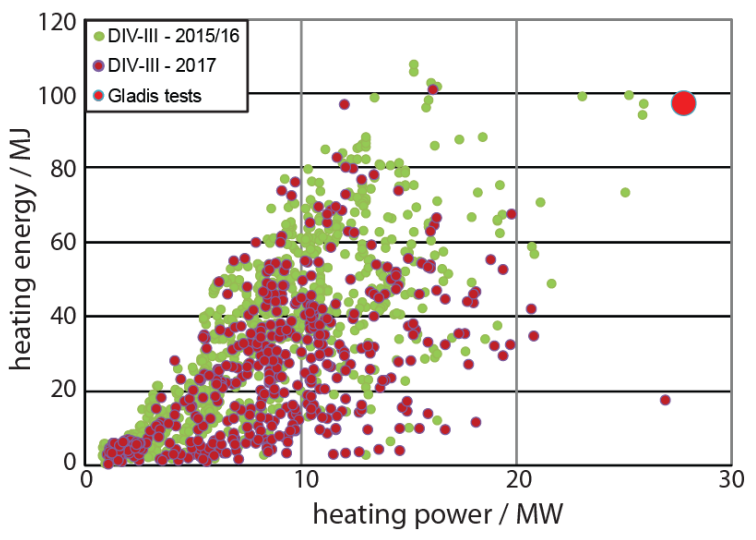

Fig. 8. Operation range for the last two experimental campaigns. At the end of 2015/16 campaign nearly all the tiles suffered from deep cracks. After 2017 campaign no deep cracks were built up. For comparison, the plasma heating power and energy equivalent to the GLADIS test is shown.

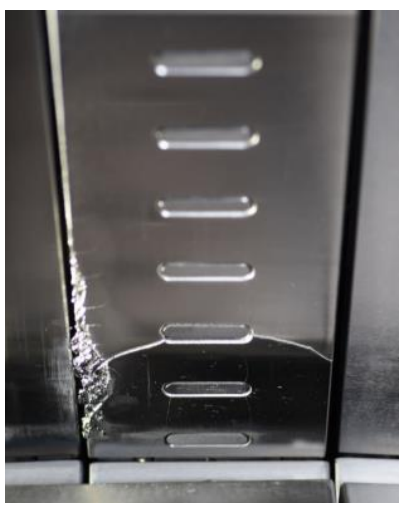

Fig. 9. Picture of a broken tile embedding Langmuir probes in AUG with strongly damaged leading edge (sector 8).

The wide tiles with titanium clamps even do not generate deep cracks, neither the wide tiles (Fig. 11) nor the split tiles.

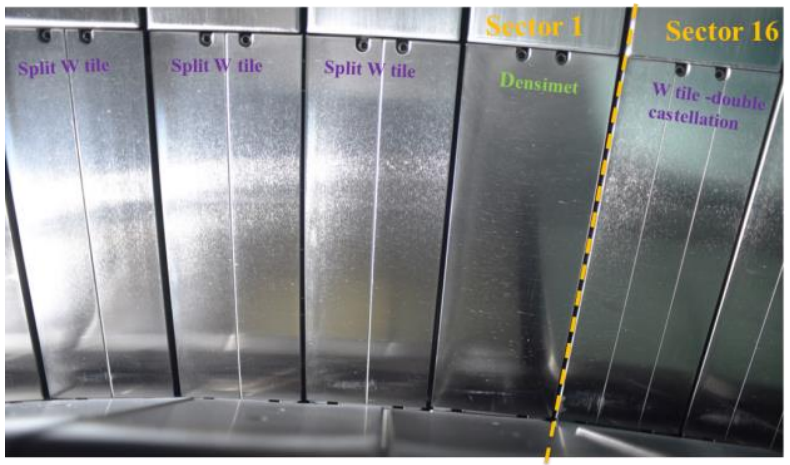

Fig. 10. Picture of half sector with standard configuration (Sector 1). On the right side, a tungsten tile with double castellations is visible (Sector 16).

Just one of the wide tiles with titanium clamp has shallow cracks in the strike line region, but, as mentioned, they are not through the tile thickness. This damaged tile, together with another wide tile were taken out from the vessel for further analyses. Both tiles have on top of the surface many tungsten droplets originating 
from the melting at the leading edge nearby (see Fig. 9). The tiles were carefully analyzed with optical microscopy: the damaged tile reveals superficial cracks disconnected from the clamping region. The cracks belonging to this network seem to be independent from each other; that would be compatible with hypothesis that the shallow cracks are induced by different shots with different strike regions. Thus, it is reasonable to think that these cracks had a thermo-mechanical origin. In Fig. 12 views of the microscopic analyses are shown. A crack width of $50 \mu \mathrm{m}$ and a crack depth of at least 100 $\mu \mathrm{m}$ were determined.

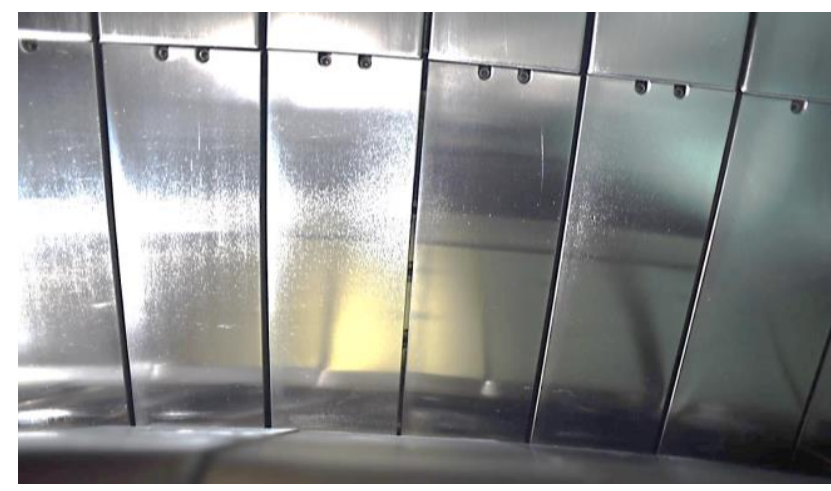

Fig. 11. Picture from AUG vacuum vessel: wide tungsten tiles with titanium clamps (Sector 7).

The microscopic analysis did not reveal cracks in the second dismounted wide tile. Apart from the droplets on surface, the tile looks like as mounted in the divertor.

It is worth to mention that a wide tile with steel clamp was temporarily mounted on the divertor manipulator [11] and it developed deep cracks after less than 100 shots (two weeks operation).

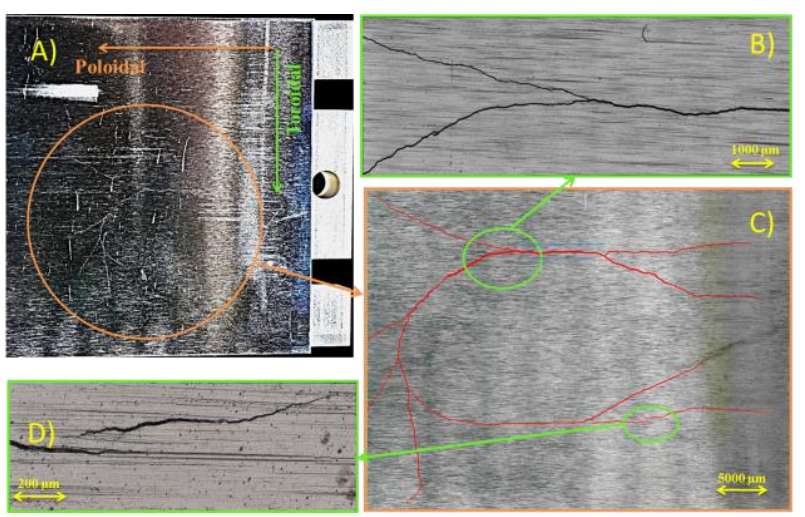

Fig. 12. On the top left: A) picture from the wide tungsten tile with titanium clamp. C) Microscopic analyses of crack path with detailed views in B) and D).

Furthermore, it should be pointed out that in 5 positions out of 13, the edges of the HPM tiles reveal signs of melting and thermally induced cracks. Since these tiles are mounted at the boundaries of the sectors, the geometrical shadowing between the neighboring tiles is critical. Laser scanning measurements reveal a reduction of the shadowing in correspondence of the melted HPM tiles. Probably disruptions or VDEs are responsible for the divertor sectors misalignments. The leading edges phenomenon is not peculiar of the HPM because the same melted edges were observed in tungsten tiles installed in the previous campaigns [7].

\section{Discussion and conclusion}

Since 2013 ASDEX Upgrade is gathering valuable experience in dealing with bulk tungsten as plasma facing component. Though the material offers a high melting temperature and low erosion rate, there is the drawback of its brittleness, which must be considered in the design of the plasma facing components. From the very beginning of AUG experiments, a problem of the formation of deep cracks was appeared: just after one week of operation in 20142 out of 128 tiles were cracked, while at the end of 2 experimental campaigns almost all the tiles were damaged. To eliminate or mitigate the deep cracks, or at least to extend the tiles lifetime, different precautions were taken, and discussed in this paper. Two proposals were considered, split and castelleted tiles, to reduce the thermo-mechanical stress and both worked nicely. The residual stresses at the tile surface exposed to the hot plasma are relieved.

On the other side, also in the sector with more flexible titanium clamps no deep cracking was observed, yet. For the first time, no deep cracks were induced in wide tiles. But the solely tile (excluding the divertor manipulator) with shallow crack belongs to this sector. From the microscopic analysis, these cracks have the characteristics of the shallow cracks: they are running superficially (few $\mu \mathrm{m}$ depth) and they appear disconnected from each other. Since they are far away from the clamping region, their origin is certainly thermo-mechanical. It is interesting to see how these cracks will evolve for a longer exposition time, if they can be considered the first stage of a deep crack or they will remain in this superficial stage. As next step, the installation of the wide tile with shallow cracks on the AUG divertor manipulator is envisaged, taking advantage of a video inspection in between shots. In this way, it would be possible to check the propagation of the cracks. The results of the intermediate inspection are overall positive: no differences between the 2 tungsten suppliers are remarkable and all the precautions definitively improve the tungsten tile behavior. The synergetic effect between thermal and mechanical loads seems to be responsible for the formation of deep cracks. The combination of the two loads accelerates the crack initiation and propagation. This statement should be confirmed by a longer exposure of the tiles in AUG, making a strict comparison between the double castellated tiles and the wide tiles with the titanium clamps. Also the performance of the HPM tiles was remarkable, especially considering that they were facing the higher induced currents and the higher thermal load due to sector misalignments. 


\section{References}

[1] I. Zammuto et al., Long term project in ASDEX upgrade: Implementation of ferritic steel as in vessel wall, Fusion Engineering and Design 98-99 (2015) 1419-1422.

[2] A. Herrmann et al., Solid tungsten Divertor-III for ASDEX Upgrade and contributions to ITER, Nuclear Fusion, 55(6) (2015) 063015 .

[3] A. Herrmann et al., Design and concept validation of the new solid tungsten divertor for ASDEX Upgrade, Fusion Engineering and Design, 88(6-8) (2013) 577-580.

[4] N. Jaksic et al., FEM investigation and thermo-mechanic tests of the new solid tungsten divertor tile for ASDEX Upgrade, Fusion Engineering and Design, 88(6-8) (2013) 1789-1792.

[5] N. Jaksic et al., Results of high heat flux tests and structural analysis of the new solid tungsten divertor tile for ASDEX Upgrade, Fusion Engineering and Design, 9899 (2015) 1333-1336.

[6] H. Greuner et al., High heat flux facility GLADIS: operational characteristics and results of the W7-X preseries target tests, Journal of Nuclear Materials, (2007) 367-370.

[7] A. Herrmann et al., Experiences with a solid tungsten divertor in ASDEX Upgrade, Nuclear Materials and
Energy (PSI2016), in press.

[8] R. Neu et al., Investigations on tungsten heavy alloys for use as plasma facing material, Fusion Engineering and Design (SOFT2016), in press.

[9] M. Li et al., Structural impact of armor monoblock dimensions on the failure behavior of ITER-type divertor target components: Size matter, Fusion Engineering and Design, (2016) 162-170.

[10] M. Li et al., Numerical design for avoiding deep cracks in the solid tungsten divertor target in ASDEX Upgrade, Unpublished

[11] A. Herrmann et al., A large divertor manipulator for ASDEX Upgrade, Fusion Engineering and Design 98-99 (2015) 1496-1499.

[12] N. Jaksic et al., Transient thermal and structural mechanics investigation of the new solid tungsten divertor tile for special purpose at ASDEX Upgrade, IEEE Transaction on Plasma Science, Vol. 42 (6) (2014) 1790-1795. 